\title{
PRIVACY ENHANCEMENT OF NODE IN OPPORTUNISTIC NETWORK BY USING VIRTUAL-ID
}

\author{
Sandeepak Bhandari and Satish Arora \\ Department of Computer Science \& Engineering, PTU University, Punjab, India
}

\begin{abstract}
An entrepreneurial system is one of the sort of remote system. Delay resistance system is correspondence organizing proposition which empowers the correspondence in such a situation where end to end way might never be exist. Message is forward on the premise of chance. Time interim to convey a message is long we can't evaluate or anticipate the time until we get the message. There is a security issue in these sorts of system. In this paper we will proposed another procedure which will expand the protection of the system and build execution of the system.
\end{abstract}

\section{KEYWORDS \\ Opportunistic Network, Architecture and Working of Opportunistic Network, Selective packet drop attack, Proposed Methodology, Simulation Results}

\section{INTRODUCTION}

A pioneering system is a class of deferral resistance system. It is shaped by the hubs having capacity to bolster this system, the hubs are joined remotely. The hubs are portable or stable so no settled framework is available in this system and this system can work even in disengaged environment. Each hub have a limited reach in which the can impart or can forward the message. A hub can forward a message just when whatever other hub comes in his extent. The hubs need to store the back rub until another hub is not come in his extent. All hubs need to work in the storeconvey forward way in this system. In this system, gathering of middle of the road hubs communicate something specific from source to destination. Hubs have no predefine topology of the system, two hub may be or never joined, no fix course between two hub is use to send message. System topology may change because of enactment and deactivation of the hub. On the off chance that destination hub is not in the scope of source hub then it passes the message to the closest hub in its extent thus on hub by hub closer to the destination. This system is anything but difficult to actualize in any circumstance or any environment like war and debacle inclined ranges where correspondence is for brief time and needs rapidly. In such environment we have less time to execute the system topology or to make a foundation. At such an area or time this system is extremely valuable to encourage the client to impart. At the point when two hubs found one another effectively then no one but they can share the message or information. A hub can trade information to its nearest hub inside of the immediate extent. At that point hubs pass the information to its nearest hub and after that the following neighbor hub store the message and sit tight for the chance to forward the message to next hub. On the off chance that a hub is deactivate because of some reason and convey the information, then at whatever point it is actuate it can continues the correspondence as entrepreneurial system is same as deferral resilience organize so time to communicate something specific is not a stress in this system the main concern is message

DOI:10.5121/ijsc.2015.6403 
is compasses to the destination. An data sprinkler is a committed and a steady hub which is altered in a devoted area in a bunch of crafty system or we can say a steady hub is available in each group of the deft system. Data sprinkler works same as different hubs in deft system it can likewise forward the message like other moderate hubs. It utilizes information sharing convention. It gather data from deft hubs that in its extent. One data sprinkler is associated with other data sprinkler (stable hub) through wired or wireless systems which have different hubs in its extent. In any case, there is an issue in the security of the entrepreneurial system. In this paper we will talked about security of the system.

\subsection{Working of Opportunistic Network}

In deft system, correspondence opportunities (contacts) are irregular, so a conclusion to-end way between the source and the destination might never exist. One of the conceivable answers for intention the above issues is to endeavours hub portability and neighbourhood sending so as to exchange information. Information can be put away and conveyed by exploiting hub portability and afterward sent amid deft contacts. The accompanying three stages show working of astute system.

1. Message sending to a middle of the road hub by source.

2. Message sending between middle of the road hubs.

3. Message sending in the middle of halfway and destination.

\section{LITERATURE SURVEY}

K. Fall (2003) observer that mobility of nodes is not a drawback, it is a technique to provide communication even in disconnected mode. In opportunistic network path between two nodes who wishes to communicate is unavailable. This network approach removes the assumption of physical end to end communication and allows nodes to forward message by a new technique store-carry-forward. Intermediate nodes can forward the message only when he gets opportunity to forward it. The message move closer to the destination when a mobile node which contains message gets an opportunity to send it. D. Nain, N. Petigara, and H. Balakrishnan (2003) studied about the Mobile Relay Protocol. MRP has been conceived to integrate pre-existing ad hoc routing protocols and manage message forwarding when no route towards the destination node of a message is found and the application that has generated the message can tolerate some form of extra delay. Messages that can be forwarded in opportunistic fashion are assigned two parameters: $x$ and $y . x$ represents the upper bound limit for the number of times the message can be relayed, i.e., the maximum number of relays that it can visit. Each time the message reaches a new relay node, $x$ is decreased by one so as to correspond to the residual number of relays that it is allowed to visit from then on. On the other hand, $y$ represents the upper bound limit for the length of a multi-hop path towards the destination node according to a traditional routing protocol. Therefore, a message can overall traverse $x$ hops over a non-connected path and $y$ hops over a connected path. S. Jain, K. Fall (2004) found that routing as a big challenge in such a network which is work even in disconnected mode and message can only forward when node get an opportunity. It is a difficult to provide an efficient routing protocol, as routing performance is depend on the network topology. But in opportunistic network, topology information is absent. A node can only find the existence of another node when the come in their communication range. In such a scenario context base knowledge is best method to design a routing protocol for this type of network.G. Ding, B. K. Bhargava (2004), in this article, five routing approaches are defined with different complexity are to enable peer to peer file sharing over MANET. Peer to peer system aims to share file without having any fixed infrastructure to a large number of users, without using any intermediate routing device, it is motivated by the file sharing application over the internet. They compare and evaluate the complexity of proposed approaches and conclude 
that the cross layer protocol perform well than simple overlaying peer to peer searching protocol in MANETs. They focus on the route discovery in this paper. They introduce five approaches to integrate the protocols of $\mathrm{P} 2 \mathrm{P}$ and MANETs.

\section{Selective PaCket Drop AtTack}

Particular Packet drop is trigger just when sticking assault falls flat. Once the bundle is normal by the bargained hub, it can look at the parcel headers, order the bundle, and choose whether to forward it or not. This activity is known as rowdiness. Post-gathering dropping is less bendy than particular sticking in light of the fact that the challenger is restricted to dropping just the parcels directed through it. Particular strategy known as the Jellyfish assault which is a traded off hub that is once in a while drops a little piece of continuous parcels and can be productively decreasing the throughput of a TCP stream to close to zero. This assault can be accomplish even by remind arbitrary postponements to TCP parcels, without dropping them, while left over convention consistent. Comparable particular dropping assaults can be develops for other system capacities, for example, the affiliation/de-relationship of STAs, and topology administration.

\section{Proposed Methodology}

The point of the actualizing protection in the entrepreneurial system is to pull in more clients to utilize this system. As protection is the fundamental concern and in this system there is not a settled foundation is available and the message is forward through numerous halfway hubs, there may be an egotistical hub which is not fascinating to forward the message to a specific destination, or the client wouldn't like to demonstrate his personality when need to impart or send message to a specific destination, then it is danger to the security of client or the bundle dropped by the childish hub. Additionally the substance of message is likewise access by the transitional hubs, so there is an issue that how to encode the message and share a key in the middle of source and destination without demonstrating to it to halfway hubs. To defeat this issue another philosophy will be proposed which confirm client with ID and Password. Along these lines a security of the system improved.

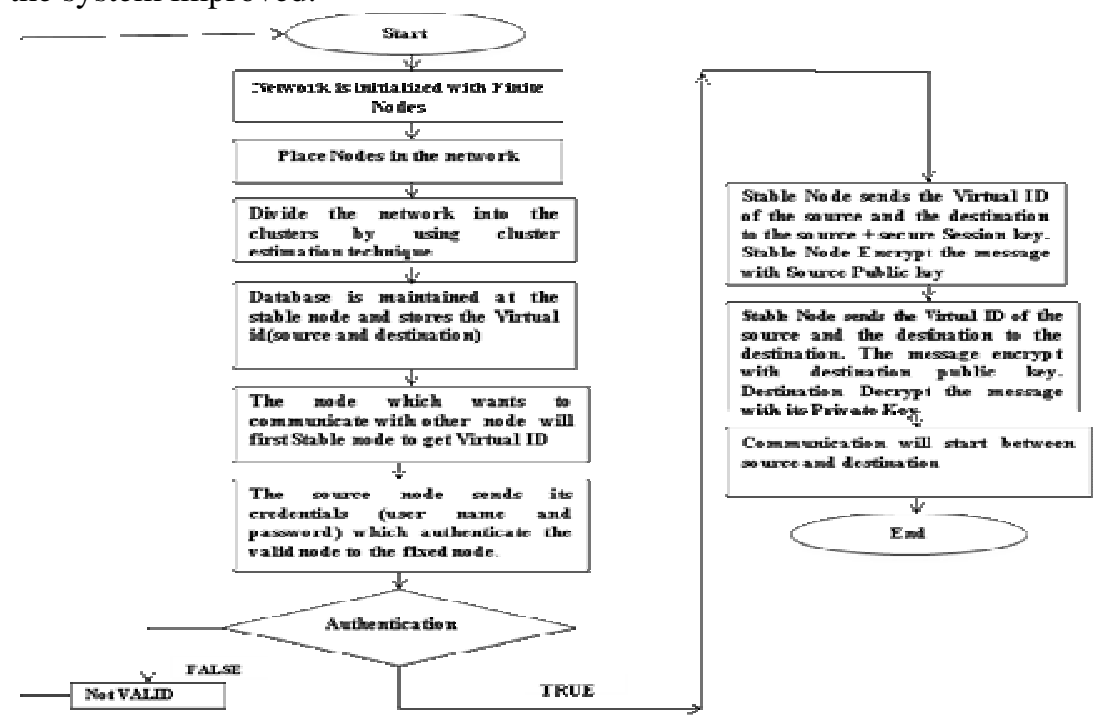

Figure 1: Proposed Methodology 


\section{Simulation SETUP}

Opportunistic remote system is executed with 13 hubs in NS2. In entrepreneurial remote system source and destination hubs are convey through different middle of the road hubs. The source hub forward parcels to that middle of the road hub which is in the scope of source hub, then halfway hub forward bundles to another transitional hub or destination hub which is in the scope of moderate hub, this procedure proceeds until bundles reach at the destination.

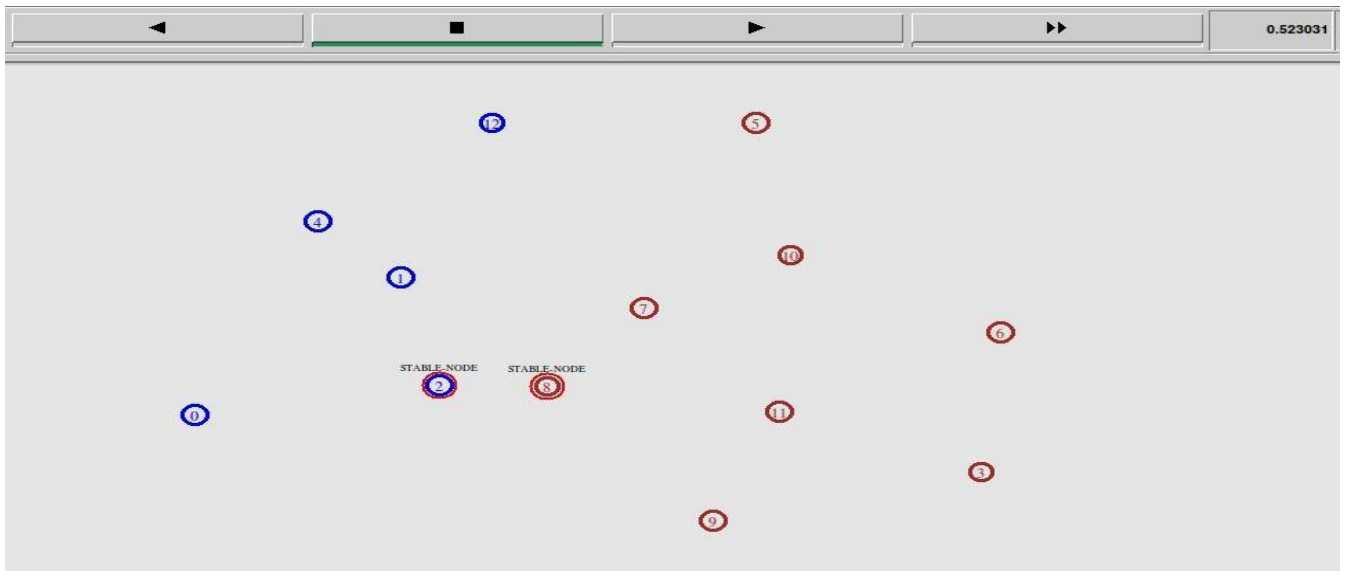

Figure 2: Opportunistic Wireless Network design.

In above figure an Opportunistic Wireless Network is configuration with 13 Nodes. It is separated into two bunches (displayed by shading). Hub 2 is the steady hub of bunch having blue shading and Node 9 is the steady hub of group having red shading. Crafty remote system is work on store-convey forward way i.e. a hub which needs to correspond with other hub it may speak with one another through different middle of the road hubs. The source hub forward bundles to transitional hub and moderate hub forward to the destination hub. In the event that hub character is appeared to all, then it causes numerous issues. A few hubs would prefer not to investigate their personality while utilizing the system. In this system the message is just forward when both hubs are in the correspondence range, so when a hub forward bundles then it area is additionally recognize such that the Node $\mathrm{x}$ and Node $\mathrm{y}$ meets at the area at a specific time. Presently for this situation transitional hub is the narrow minded hub, which check the destination address and not wishes to forward the bundles. Presently for this situation middle of the road hub is narrow minded hub, which check the destination address and not wishes to forward the bundles. Middle of the road hub drops the parcels of the destination hub henceforth correspondence is not finished in the middle of source and destination.

For giving secure correspondence in the middle of source and destination hub, two systems are utilized to be specific verification and Virtual-ID. Validation instrument is utilized for verify substantial hub of bunch and Virtual-ID is utilized for protection of hub, which conceal the area of hub. The hub which needs to impart to the next hub will first correspond with the settled hub to get the virtual ID. At the point when a hub needs to send message, it first sends a solicitation message to the steady hub to get a virtual ID. This message is forward by the middle of the road hubs to the steady hub. In the solicitation message source hub send its NODE_ID and PASSWORD which is use by the steady hub to verify the substantial hub of the group. When the source hub verifies the hub then stable hub correspond with the steady hub of destination bunch and trade data and overhaul their table. Presently steady hub of source group will sends another virtual ID to the source, new ID of destination and a session key with which the source hub encode the message. This message is presently scramble by general society key of the source 
hub. Furthermore, stable hub additionally sends another virtual ID to the destination, virtual id of source hub and session unscrambling key. This message is encoded by people in general key of destination hub.

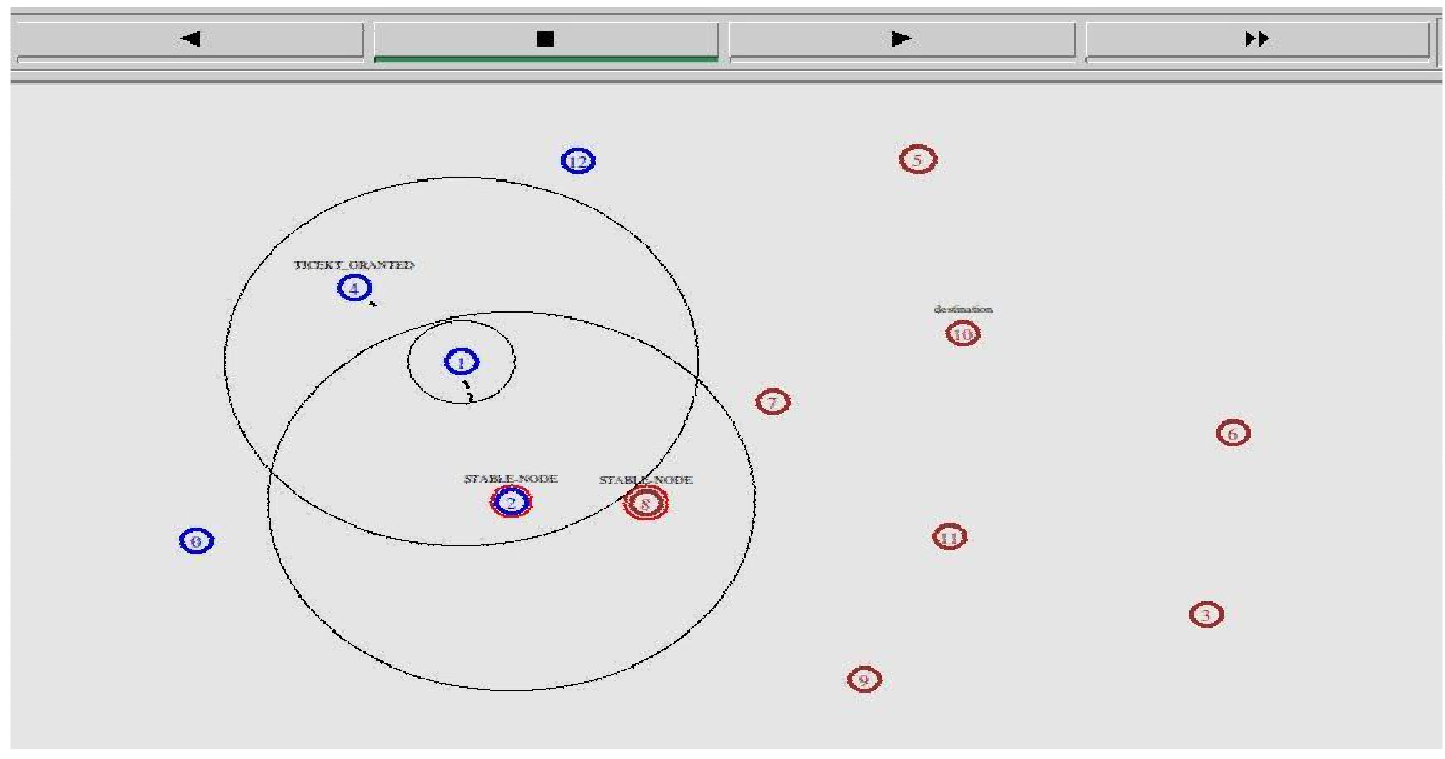

Figure 3: Authentication by stable node.

In above figure stable (hub 2) validates the hub 4 as a substantial hub of bunch. Stable hub verifies the hub by coordinating its NODE_ID and PASSWORD.

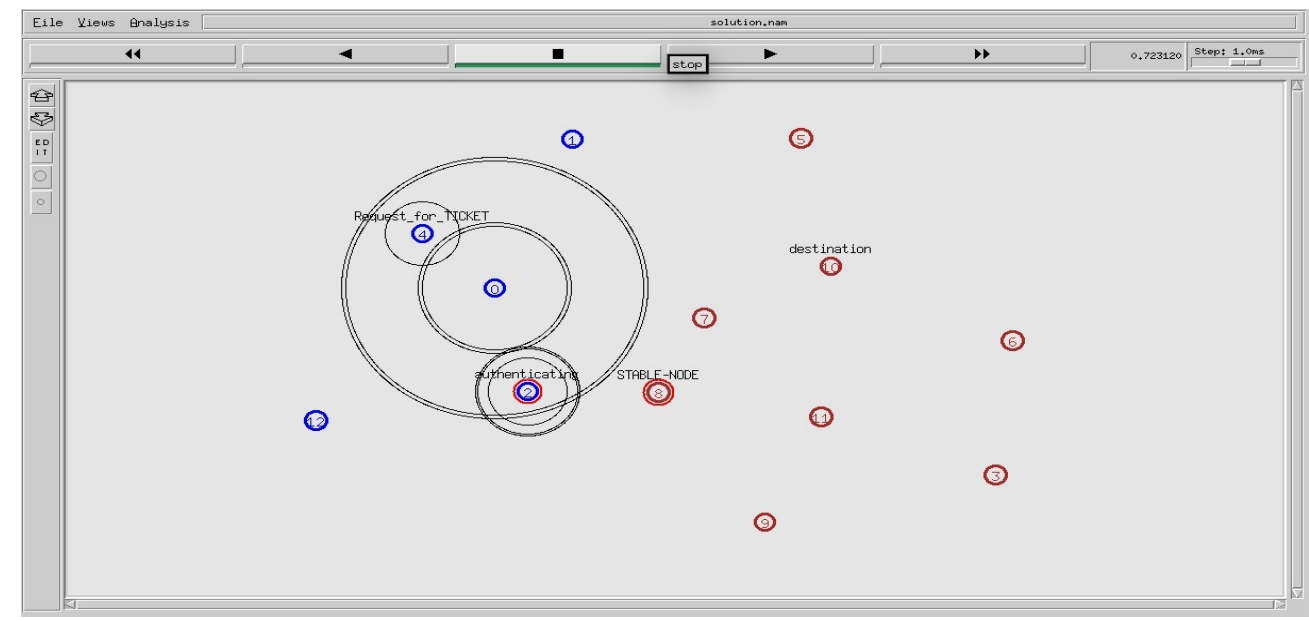

Figure 4: Ticket Receiving by the Source Node

In above figure stable hub sends ticket to the source hub after confirmation and imparting data to the steady hub of bunch in which destination is present.Ticket contains taking after:

- A new virtual-ID of Source Node.

- A new virtual-ID of Destination Node.

- A session key to encode the message. 
Virtual-ID is utilized for conceal the character of hub which is utilized to improve the protection of node.The personality of hub is uncovered when two hubs accompanies in scope of one another by trading parcels with one another so when a hub forward bundles then it area is likewise distinguish such that the Node $\mathrm{x}$ and Node $\mathrm{y}$ meets at the area at a specific time. A middle of the road hub is egotistical hub, which check the destination address and not wishes to forward the packets.To conceal the personality of hub, stable hub allots virtual-id to source hub which is just known by source hub, destination hub and stable hub itself. Presently when source hub send bundles to halfway hub it send its virtual-id and virtual-id of destination hub alongside parcels alloted by stable hub and virtual-id is dependably time distinctive when correspondence begin in the middle of source and destination so it is unrealistic for moderate hub to recognize the personality of hub in light of the fact that just stable hub gift virtual-id to source hub and destination hub and which specific virtual-id is appoint to a specific hub for specific time of correspondence. Along these lines, as number of secured parcels is expanded the more protection is upgraded on the grounds that character of hub is uncovered through bundles transmission between nodes.Now Source hub utilized new Virtual-ID for correspondence with destinatio

\section{Simulation RESULTS}

In this segment the execution of astute system is broke down with upgraded method and existing procedure. The execution of system is analyzed on four parameters to be specific Throughput, Packetloss,Delay and Privacy.

The X-hub speak to the recreation time in seconds and Y-hub speak to Number of Packets.

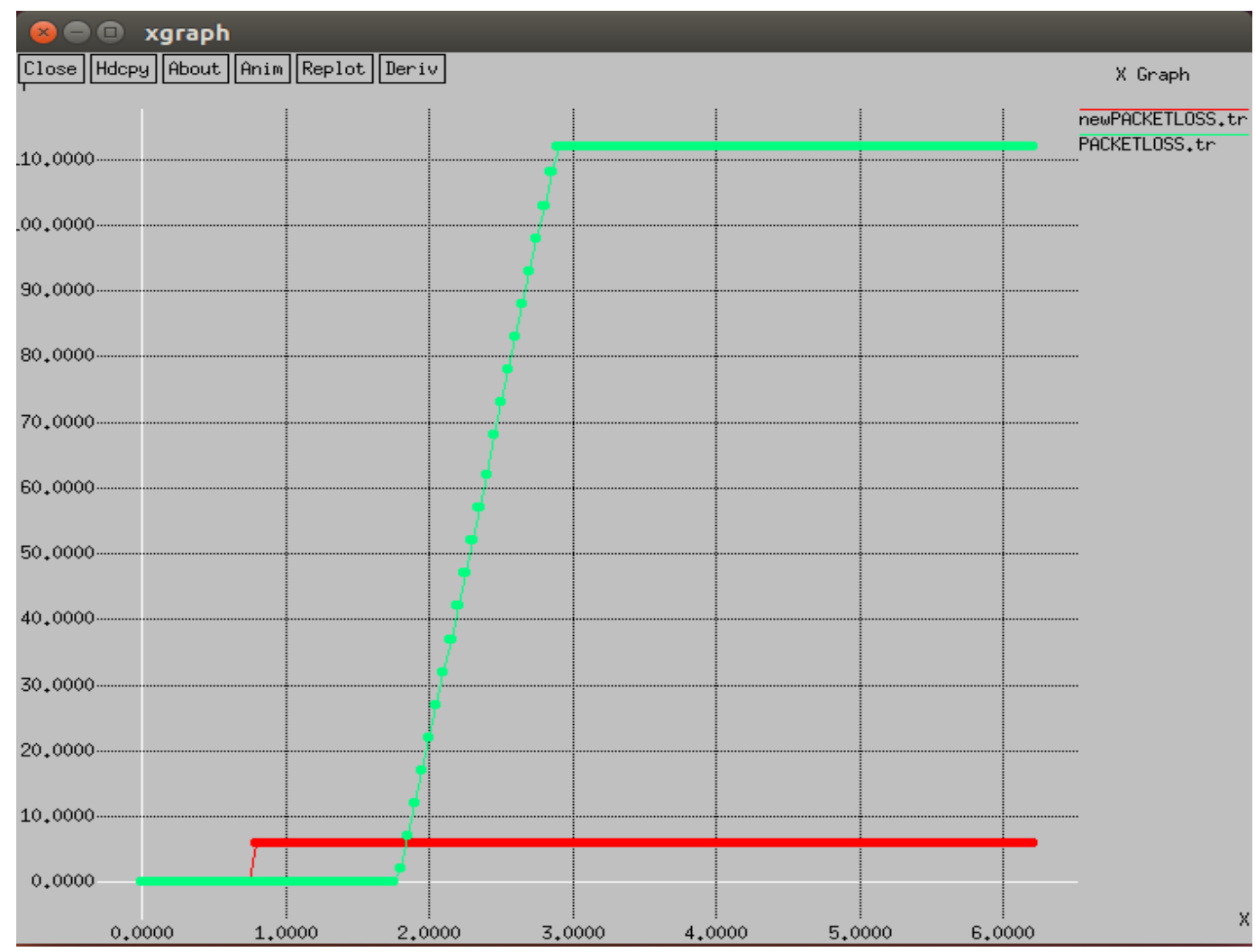

Figure 5: Packet loss Graph 
The above figure demonstrates the examination of both systems as far as bundle misfortune. The outcome demonstrates that improved instrument lessened the considerable quantities of bundle misfortune in sharp remote system as contrast and existing component. The Y-hub speak to number of bundles and X-hub speak to time in seconds.

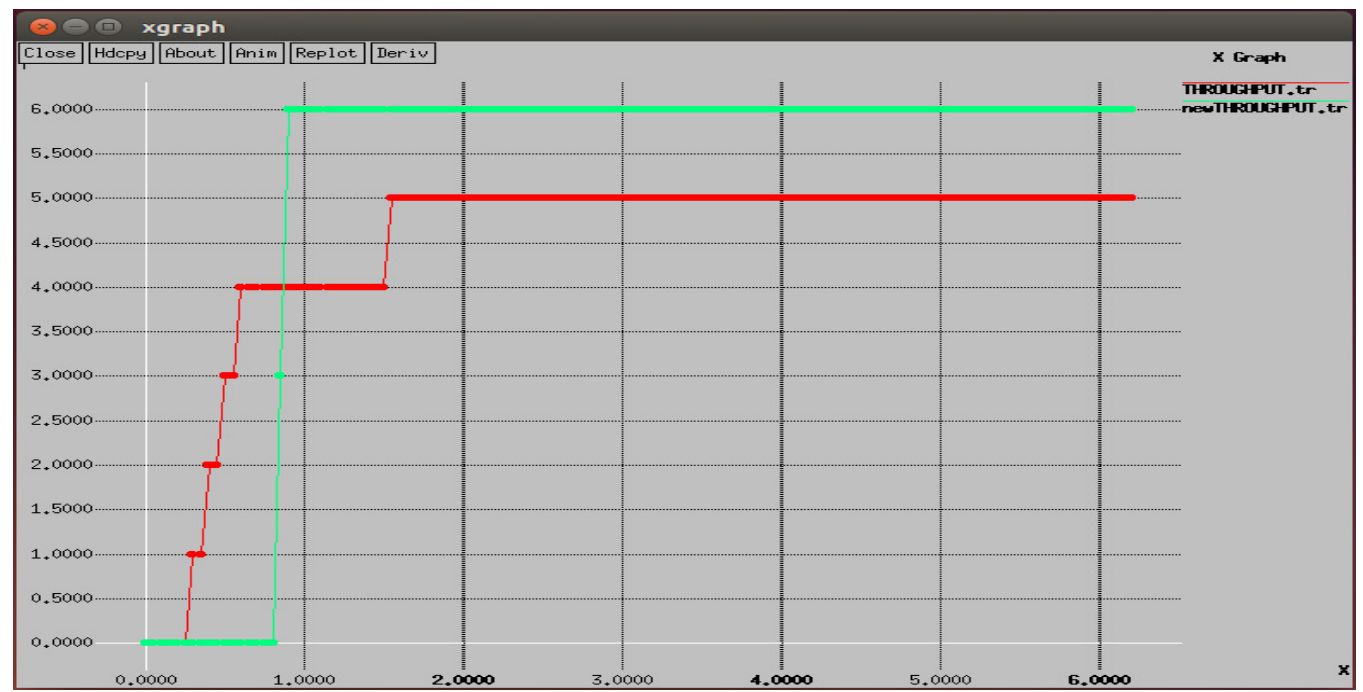

Figure 6: Throughput Graph

The above figure demonstrates the correlation of throughput of both components. The above chart demonstrates that upgraded component enhance the throughput of astute remote system. The Y-hub speak to number of parcels and X-hub speak to time in seconds.

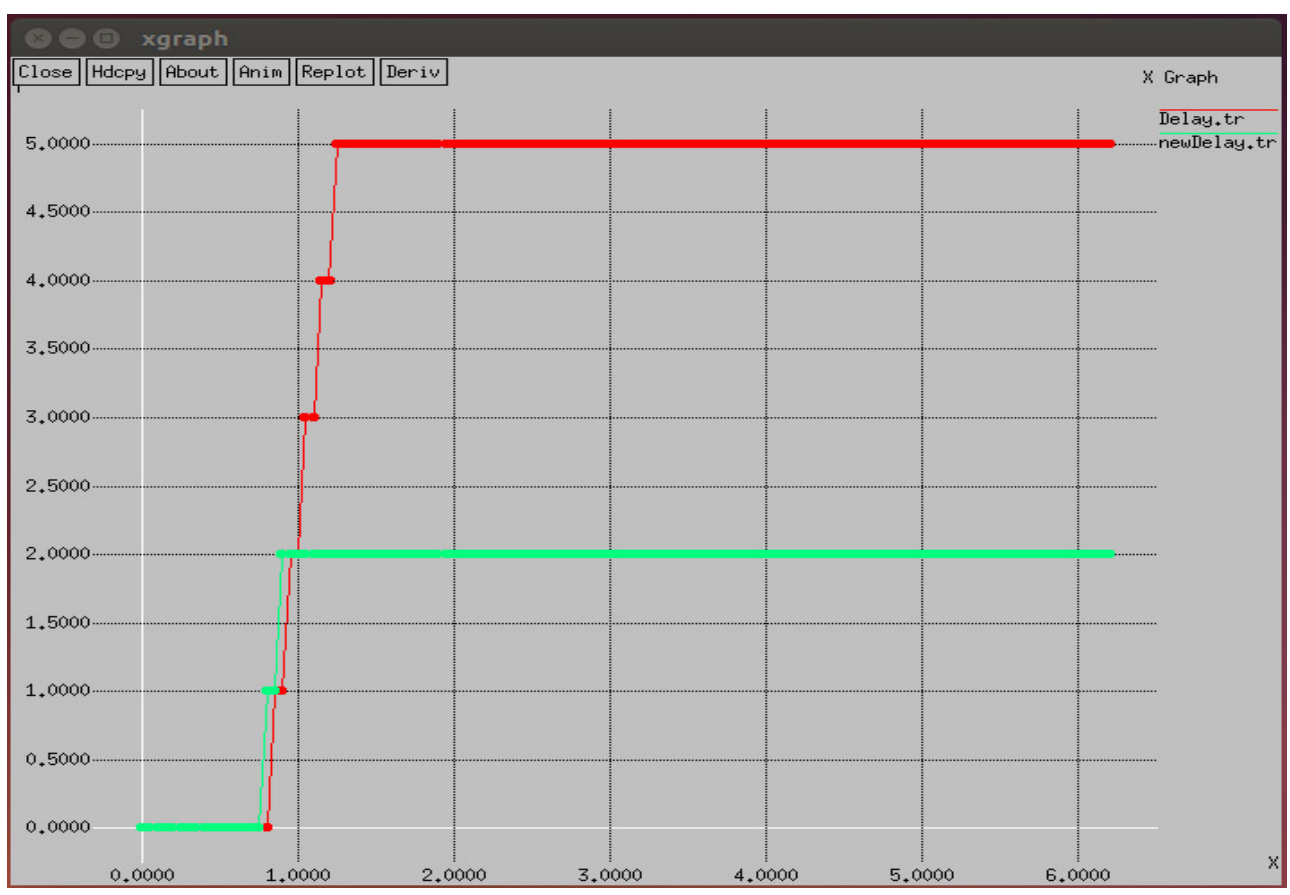

Figure 7: Delay Graph 
The above figure demonstrates that improved instruments extraordinarily diminished the postponement of bundles in shrewd when contrasted with existing component. The Y-hub speak to number of parcels and $\mathrm{X}$-pivot speak to time in seconds.

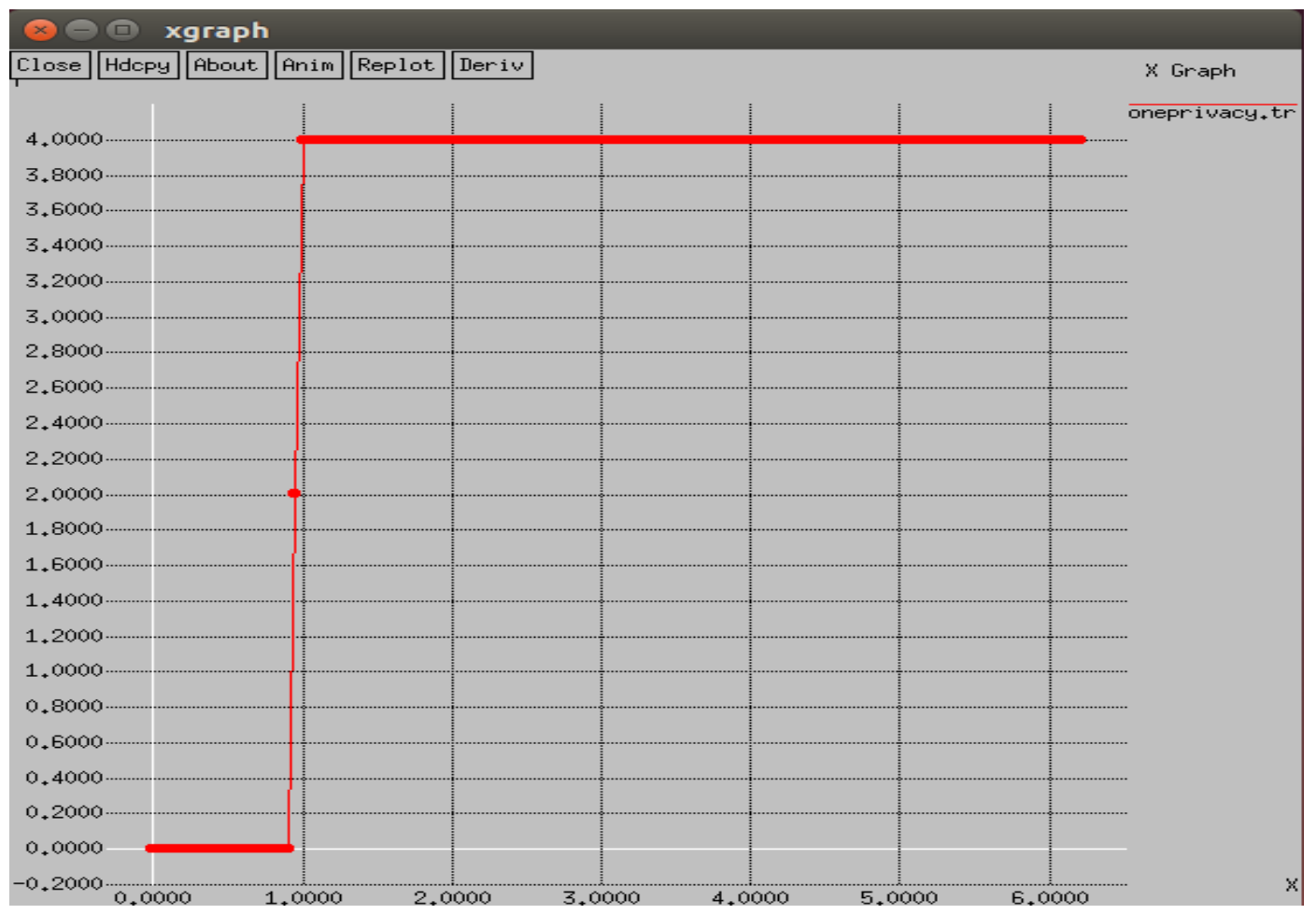

Figure 8: Privacy Graph

The above chart demonstrates the protection of hub in astute remote system is improved with upgraded component. The Y-pivot speaks to the quantity of secure bundles and X-hub speak to the recreation time

\section{CONCLUSION}

In this examination we find that sharp system is exceptionally valuable if security is kept up. We propose a system construction modeling in which a hub need to make an impression on a destination and he wouldn't like to investigate his way of life and also destination personality then he first correspond with stable node(trusted hub) and get a virtual id for a period a period. Stable hub go about as exceptional hub, which contains data of each hub of the bunch and confirm the hubs who wishes to impart and give virtual id to that hubs. Furthermore a session key is given to encryption of message to the source and unscrambling key to the destination for keeping up the secrecy of the message. This methodology gives protection to the client and decreases the parcel misfortune by a narrow minded hub. What's more, the charts speak to the adjustment in parcel misfortune and throughput of pioneering system.

\section{FUTURE SCOPE}

In our work we reason a method to give security to a client in deft system on the premise of giving virtual id by a steady hub, which is available in each bunch. Be that as it may, this system expands the work heap of a sender who wishes to convey. In future we attempt to discover a way 
which diminishes the client work by giving some new instrument to conceal the id of client. Because of foundation less building design and versatility of the hubs, sharp system faces numerous issues identified with security, protection, hubs verification and proficient steering convention.

\section{REFERENCES}

[1] Jia Jianbin, Chen Yingwen, Xu Ming, Xia Geming, and Xiao Xiaoqiang,(2013) "Towards the Benefit of Multi-Hop Relaying in Opportunistic Networking",IEEE International Conference on Green Computing and Communications and IEEE Internet of Things and IEEE Cyber, Physical and Social Computing ,pp.658-662.

[2] Augustin Chaintreau, Pan Hui, Jon Crowcroft, Christophe Diot, Richard Gass, and James Scott,(2006) “Impact of Human Mobility on the Design of Opportunistic Forwarding Algorithms", IEEE Infocom. IEEE Computer Society

[3] Papaj Jan, Dobos Eubomir and Cumar,(2012)“ Opportunistic Networks and Security”Journal of Electrical and Electronics Engineering, vol. 5,no.1.

[4] L. Lilien, Z. H. Kamal, V. Bhuse, and A. Gupta,(2006) "The Concept of Opportunistic Network and their Research Challenges in Privacy and Security","Mobile and Wireless Network Security and Privacy", Book Chapter, pp. 85-117.

[5] L. Pelusi, A. Passarella, and M. Conti,(2006) "Opportunistic Networking: data forwarding in disconnected mobile ad hoc networks", IEEE Communications Magazine, vol. 44, no.11, Nov.

[6] D. Nain, N. Petigara, and H. Balakrishnan,(2003) "Integrated Routing and Storage for Messaging Applications in Mobile Ad Hoc Networks", in Proceedings of WiOpt, Autiplis, France, March.

[7] S. Jain, K. Fall and R. Patra,(2004) "Routing in a delay tolerant network", Proceedings of the ACM Conference on Applications, Technologies, Architectures, and Protocols for Computer Communications, pp.145-158.

[8] Abdullatif shikfa, Melek Onen and Refik Molva(2010),"Privacy and Confidentiality in context based and epidemic forwarding" published by Elsevier,pp.1493-1504.

[9] Abdullatif shikfa,Melek Onen and Refik Molva,(2012)“ Local key management in Opportunistic network".International Journal Networks and distributed Systems, Vol.9,Nos.1/,pp.97-116.

[10] B.Poonguzharselvi1 and V.Vetriselvi,(2012) "Trust Framework for Data Forwarding in opportunistic Network Using Mobile Traces”,International Journal of.Wireless Networks Vol.4,No.6.

[11] K. Fall,(2013) “ A delay-tolerant network architecture for challenged internets”, Paper presented in the Proceedings of the ACM Conference on Applications, Technologies, Architectures, and Protocols for Computer Communications , pp.27-34.

[12] Xingguang Xie, Yong Zhang, Chao Dai and Mei Song,(2011)"Social relationship enhanced predicable routing in opportunistic network" Seventh International Conference on Mobile Ad-hoc and Sensor Networks.

[13] L. Dora, T. Holczer ,Hide and Lie,"Enhancing(2010) Application-level Privacy in Opportunistic Networks",Proceedings of the Second International Workshop on Mobile Opportunistic Networking ,pp.135-142..

[14] G. Costantino ,F. Martinelli and P.santi,(2012)“ Privacy-preserving interest-casting in opportunistic networks",IEEE wireless communications and networking conference: mobile and wireless networks.

\section{AuTHORS}

Sandeepak Bhandari seeking his Master of Technology in C.S.E from P.T.U University, Punjab, India. This paper portrays the examination work Which he finished amid his expert of innovation. 